\title{
Progress in orthopedic biomaterials and drug delivery
}

\author{
Blanka Sharma $^{1} \cdot$ Shyni Varghese ${ }^{2}$
}

Published online: 2 March 2016

(C) Controlled Release Society 2016

Musculoskeletal conditions, including arthritis, back pain, and skeletal injuries/trauma, affect $50 \%$ of the adult population in the U.S.A., and account for the second greatest cause of disability worldwide (www.bjdonline.org). The aggregate total annual cost of musculoskeletal diseases was $\$ 796$ billion in 2011. While most musculoskeletal injuries and diseases have low mortality, they have a tremendous impact of quality of life and morbidity.

Biomaterials have a long history in orthopedics - metal and plastic implants have been used over the last 60 years to treat end-stage joint degeneration and skeletal tissue loss, and alleviate the resulting pain and disability. First-generation orthopedic implants were designed to be biologically inert and selected primarily for their mechanical properties. However, they do not replicate the function of normal tissue and have a limited life span in the body, which is especially problematic in younger patients. Recent decades have focused on the development of biomaterials that are biodegradable and incorporate tissue-specific physicochemical cues to guide cell processes and tissue morphogenesis, with the goal of restoring natural tissue structure and function. These advances in biomaterials provide critical enabling technologies for the delivery of stem cells, gene therapies, and new biologics in the orthopedic field. This special issue highlights the recent advances and future directions in orthopedic biomaterials and

Blanka Sharma

blanka.sharma@bme.ufl.edu

1 J. Crayton Pruitt Family Department of Biomedical Engineering, University of Florida, Gainesville, FL, USA

2 Department of Bioengineering, University of California, San Diego, CA, USA drug delivery systems, and showcases emerging pharmaceutical and regenerative approaches to treat injuries, diseases, and disorders of the musculoskeletal system.

Non-union bone fractures are a significant clinical challenge, and bone tissue engineering could have a key impact in improving current clinical options. Telvin et al. review the application of stem cells from various sources towards bone tissue engineering. The ability of transplanted stem cells to survive, continue to differentiate, and form bone tissue is key to making stem cell-mediated bone tissue regeneration approaches successful. The research article by Wen et al. precisely address this challenge. Their study compares the in vivo bone tissue forming ability of human bone marrow derived stem cells and human embryonic stem cells using osteoinductive mineralized materials as a scaffold. The article by Nyberg et al. reviews progress and challenges in achieving precise temporal and spatial control of growth factor presentation for functional bone tissue engineering ex vivo and in vivo. Ultimately, successful bone regeneration requires integration between the repair and native bone tissue. Botchway and colleagues address this critical issue, by modifying the surface of a bone allograft with FTY720, a synthetic analog of sphingosine 1-phosphate, to promote endogenous bone formation and osseous integration at the interface. Another key hurdle for the success of bone tissue engineering is vascularization in vivo. The review by Garcia and Garcia summarizes the current strategies and new developments targeted at vascularization of engineered bone, including growth factor and cell delivery, gene therapy, biomaterials, and combination approaches. The article by Tonello et al. demonstrates the potential of human placental matrix for promoting angiogenesis and its controlled release from biodegradable microparticles.

In addition to bone, this special issue contains articles that highlight advances in cartilage tissue regeneration and osteoarthritis (OA) therapies. Osteoarthritis is the leading cause of 
disability in the U.S.A., marked by degeneration of the articular cartilage, and has no cure. The review by Kavanaugh et al. summarizes emerging particle based technologies for the detection and treatment of OA that could lead to diseasemodifying therapies for OA that are currently lacking in clinical practice. The review Orthopedic Tissue Regeneration: Cells, Scaffolds, and Small Molecules describes technologies at the forefront of cartilage regeneration, in addition to bone, and the how cross-talk between the cartilage-bone interface affects joint preservation and disease. Riboflavin induced photo-crosslinking of collagen hydrogel and its application in meniscus tissue engineering by Heo et al. addresses meniscal damage, a common knee injury that leads to posttraumatic OA.

This issue has assembled leaders in nanomedicine, tissue engineering, and drug delivery, covering topics such as vascularization strategies, cell-based therapies and stem cell recruitment, novel drug delivery systems, and next-generation biomaterials for the regeneration of musculoskeletal tissues. 